\title{
Translational research: Lessons from past research, growing up nowadays, and development goal in future
}

\author{
ZHANG ZhuQin, CHEN HouZao \& LIU DePei* \\ National Laboratory of Medical Molecular Biology, Institute of Basic Medical Science, Chinese Academy of Medical Sciences and Peking \\ Union Medical College, Beijing 100005, China
}

Received October 19, 2011; accepted November 10, 2011

Citation: Zhang Z Q, Chen H Z, Liu D P. Translational research: Lessons from past research, growing up nowadays, and development goal in future. Sci China Life Sci, 2011, 54: 1085-1088, doi: 10.1007/s11427-011-4252-0

Recently, with coming of the "omics" era and rapid development of basic research in biology and medicine, huge information about biology and life has been achieved. However, many research results cannot be translated into clinical practice. Under this circumstance, the concept of "translational research" is raised [1-4] and widely spread. "Translational research" is patient-oriented research which connects basic medicine and clinical medicine [2]. It means more directly and quickly raising questions from clinical practice to basic research and translating findings in basic research into clinical practice. The process of translational research can be summarized as follows: first, according to clinical practice, raising clinical problems and refining scientific questions; then, systematic and in-depth research, which integrates diverse disciplines, including epidemic study, basic research and drug discovery, is carried out; last, through research, the potentially effective strategies or methods for diagnosing, treating or preventing diseases are achieved and translated into clinical practice. Translational research has been developed rapidly in recent years. It is used to direct research in cardiovascular diseases (CVD), cancer, infectious diseases, metabolic diseases, etc.

Though the concept "translational research" is relatively new, the bedside-to-bench-to-bedside translational strategy is not original. In the past century, especially in the past several decades, many successful studies were done. These studies contributed to human health greatly and are good

*Corresponding author (email: liudp@pumc.edu.cn) examples for translational research. In this review, we will choose to talk several of these studies. We hope that these successful examples in the past will facilitate us to deeply understand translational research in the future. We will talk about research in CVD which achieved great progress in the past.

In the mid-20th century, CVD was severe and almost accounted for half of all death in the USA [5]. Framingham Heart Study, an epidemiologic study, was carried out to study CVD in the town of Framingham, Massachusetts in 1948, with 5209 adult healthy subjects enrolled [6]. The detailed information for each subject, including diet, exercise, social relationship, physical examination and medical history, was recorded [6]. The study was implemented for about 20 years at its first stage. After that, the second generation and the third generation of subjects were enrolled in 1971 and in 2002 respectively [7,8]. Up to now, the study has lasted more than 60 years. The results from Framingham Heart Study showed that several factors, including high LDL cholesterol level, cigarette smoking, hypertension, obesity, diabetes, menopause, increased the risk of heart diseases, whereas exercise and high HDL cholesterol decreased the risk of heart diseases. It also showed that the social and physiological factors influenced CVD [9-12]. In recent years, with the development of technology in genetics, such as GWAS technology, some genetic factors contributing to CVD, such as chromosome region 9p21 [13,14], were found. Therefore, overall, from Framingham Heart Study, the causes for CVD, including many environmental 
and genetic factors, were found. Framingham Heart Study changed people's ideas about CVD, showing that the disease is preventable. It introduced the term "risk factor" [15], which is used widely nowadays. Framingham Heart Study is famous for its long duration and outlook and is thought of a very successful research program.

Another research about how statin was developed provides a good example for drug discovery for treating CVD. More than 100 years ago, it was found that there was cholesterol accumulation in the artery walls of people that died from myocardial infarction [16]. The later Framingham heart study also showed that there was positive correlation between total blood cholesterol level and CVD [17]. Biochemical analysis revealed that cholesterol was derived from diet or synthesized by human body, so inhibition of cholesterol synthesis was a target to lower cholesterol level without modifying diet. In early studies, Triparanol, which inhibits an enzyme involved in the later stage of cholesterol synthesis, was discovered and introduced into clinical trial [18]. However, because inhibition of the enzyme led to accumulation of its substrate desmosterol and caused severe side effect, Triparanol was withdrawn [19]. HMG-CoA reductase (HMGR), the rate-limiting enzyme for in vivo synthesis of cholesterol, was another potential target. Since inhibition of HMGR did not cause accumulation of its substrate hydroxymethylglutarate which is water soluble and could be metabolized through another pathway, HMGR was attractive as a target [16]. In the 1970s, one group found the first product inhibiting HMGR in a fermentation broth of Penicillium citrinum, with the idea that some microorganisms would produce compounds to fight against other organisms that utilize sterols or other isoprenoids for growth [20]. Later, another product in a fermentation broth of Aspergillus terreus was found [21], which is currently known as lovastatin and is widely used. Nowadays, there are diverse effective statin drugs for treating CVD [16]. Statin was reported to reduce the risk of heart attacks and strokes by about one quarter to one third [22-24] and was among the most prescribed drug in the world today.

In addition to epidemic study and drug discovery, some other techniques were also developed to treat CVD. As we know, CVD is often caused by occlusion of coronary artery. Atherosclerosis is the major reason for occlusion. The severe atherosclerotic plaques, especially those unstable atherosclerotic plaques, are susceptible to rupture and cause thrombosis, leading to narrow or occlusion of artery and myocardial infarction. For those patients who have already had myocardial infarction, or, for those patients who are very susceptible to myocardial infarction, taking statins only is not much effective. Percutaneous coronary intervention (PCI), one medical operation, was developed to treat the occlusive (narrowed) coronary arteries. It was first developed in 1977. By the mid-1980s, many leading medical centers throughout the world were adopting the procedure as a treatment for coronary artery disease (CAD). Now, PCI is widely used in clinical practice. The usage of PCI significantly decreased mortality of CVD [25].

Overall, from Framingham Heart Study to the development of statin to PCI treatment, many successful translational studies in CVD have been done. These examples are all good examples of patient-oriented "Bedside to Bench to Bedside" translational research.

Nowadays, CVD is still severe. It is estimated that CVD accounts for $29 \%$ of all-cause death in the world nowadays [26]. In China, it is estimated that the number of patients with CVD is 230 million, which means 2 out of 10 adults are afflicted with CVD [27]. However, in clinical practice, there are many limits to the currently available clinical methods. Firstly, for some patients, statin treatment may cause mild to severe side effects, including muscle pain, muscle damage and liver damage. Thus, those patients cannot tolerate statin treatment. Secondly, for those patients taking PCI, restenosis often occurs after several months, so other effective drugs or methods preventing restenosis are urgently needed. Thirdly, the economic cost for treating or curing CVD nowadays is still very high. For these reasons, it is important to create more convenient and low cost methods or strategies to prevent CVD.

Recently, calorie restriction was found to be a promising and practical strategy for preventing CVD. Calorie restriction means reducing total calorie intake to $60 \%-70 \%$ of that from usual diet without malnutrition [28,29]. One long-term report in rhesus monkey shows that, compared to normal diet, calorie restriction reduces incidence of aging associated CVD by a half [30]. Calorie restriction completely prevents aging associated glucoregulatory impairment which is highly incident in normal diet [30]. In human, calorie restriction was reported to reduce risk factors for CVD such as total cholesterol, LDL cholesterol, triglyceride, fasting glucose, fasting insulin, CRP and blood pressure, but increased beneficial factors such as HDL cholesterol [31]. More directly and convincingly, calorie restriction was shown to reduce carotid artery intima medium thickness (IMT), which is an indicator of atherosclerotic plaque [31]. Therefore, calorie restriction is very effective in preventing CVD. Calorie restriction is easy to operate. It provides a convenient and effective method to promote health and prevent diseases. Metabolism is the basic characteristic of human body. Calorie restriction may change the status of human body through changing metabolism, and, while short term calorie restriction leads to subtle quantitative change of metabolic profile, long term calorie restriction may result in great qualitative change of metabolic profile, shifting the human body from a status of calorie accumulation to a status with appropriate calorie.

The role of calorie restriction for treating diseases and its molecular mechanisms were widely studied in recent years. SIRT1, an NAD dependent class III deacetylase, is important for mediating effect of calorie restriction [28,32,33]. There are diverse beneficial effects for SIRT1 in preventing 
atherosclerosis. SIRT1 upregulates eNOS expression but inhibits p66shc gene expression in endothelial cells, both of which prevent endothelial dysfunction [34,35]. PGE2 and COX2 are two molecules promoting inflammation and atherosclerosis formation. In macrophages, SIRT1 inhibits COX2 expression and PGE2 production and inhibits inflammation [36], and also decrease Lox-1 mediated foam cell formation [37]. Neointima formation is a common event after intervention therapy in clinical practice. In mice, SIRT1 was found to inhibit neointima formation by suppressing smooth muscle cell proliferation and migration [38]. Thus, SIRT1 may be a target for preventing neointima formation in human after PCI operation.

To promote translational research, it is required to set up translational research centers and cultivate a large number of talents who are engaged in translational research. Today, many translational research centers have been built up and numerous translational research programs have been carried out. These centers provide good hardware and the programs offer good human resources for translational research.

In China, many translational research centers have been built or are under construction. Specific examples for translational research centers which have been built include Translational Medicine Center of Chinese Academy of Medical Sciences and Peking Union Medical College in Beijing, Northeast Clinical and Translational Medicine Center in Harbin, and many translational medicine centers in other major cities, including Shanghai, Hangzhou, Guangzhou, Changsha and Nanjing. Some centers are under construction.

Currently, there are some programs available for translational research in China. First, there are eight-year programs for medical students in some medical schools, including Peking Union Medical College and others. After clinical training, students are given eight-month research training, where they could learn theories, techniques for basic research and do some research. Therefore, these students have good background for both clinical research and basic research. Second, China has a large number of medical graduates who received five-year programs. Some of them passed examinations and go to research institutes or schools as masters to engage in basic research. These graduate students, with good background for clinics, provide potential human resource for translational research. In China, current government supports for translational research include funding of the National Natural Science Foundation of China for applied medical research, broad support for science and technology projects from the National Basic Research Program of China (973), and Science and Technology Special Projects support for research in drug discovery and infectious disease.

Translational research needs "translational integration" of multidisciplines, including epidemiology, basic medicine, drug discovery and new techniques. It should promote exchanges and cooperations among different disciplines and build platforms to integrate hardware, software and talents. Today, with the coming of globalization and development of information technology, exchanges among people are becoming more feasible, frequent and effective than ever. Cooperation in a wide range is possible. Some national programs to promote translational research in China, such as Key Drug Innovation Program, are carried out. As an international cooperation, Sino-US Translational Research Symposium was held in Beijing in 2010 and in Shanghai in 2011. These programs greatly promoted translational integration from different disciplines.

The set up of translational research centers, the cultivation of translational research talents, and the cooperation among different disciplines, all put forward the rapid development of translational research nowadays. With the development of translational research in CVD or in other diseases, such as cancer, more and more strategies and methods for preventing, diagnosing or treating diseases will be achieved and benefit human health.

The ultimate goal for translational research may be to realize "9P" medicine, which includes protecting health, promoting health, prolonging healthy life span; predicting diseases, pre-warning diseases, preventing diseases; population policy, personal participation, and personalized medicine. Among these, prolonging healthy life span, preventing diseases and personalized medicine may be the most important and can be called " $3 \mathrm{P}$ " medicine. The "9P-3P" medicine will guide the development of future translational research.

1 Geraghty J. Adenomatous polyposis coli and translational medicine. Lancet, 1996, 348: 422

2 Marincola F M. Translational medicine: A two-way road. J Transl Med, 2003, 1: 1

3 Zerhouni E A. Translational and clinical science-time for a new vision. N Engl J Med, 2005, 353: 1621-1623

4 Zerhouni E. Medicine. The NIH Roadmap. Science, 2003, 302: 63-72

5 Fox C S, Evans J C, Larson M G, et al. Temporal trends in coronary heart disease mortality and sudden cardiac death from 1950 to 1999: the Framingham Heart Study. Circulation, 2004, 110: 522-527

6 Dawber T R, Meadors G F, Moore F E Jr. Epidemiological approaches to heart disease: the Framingham Study. Am J Public Health Nations Health, 1951, 41: 279-281

7 Kannel W B, Feinleib M, McNamara P M, et al. An investigation of coronary heart disease in families. The Framingham offspring study. Am J Epidemiol, 1979, 110: 281-290

8 Splansky G L, Corey D, Yang Q, et al. The Third Generation Cohort of the National Heart, Lung, and Blood Institute's Framingham Heart Study: design, recruitment, and initial examination. Am J Epidemiol, 2007, 165: 1328-1335

9 Castelli W P. Epidemiology of coronary heart disease: the Framingham study. Am J Med, 1984, 76: 4-12

10 Wilson P W, D'Agostino R B, Levy D, et al. Prediction of coronary heart disease using risk factor categories. Circulation, 1998, 97: $1837-1847$

11 Gordon T, Kannel W B, Hjortland M C, et al. Menopause and coronary heart disease. The Framingham Study. Ann Intern Med, 1978, 89: 157-161

12 Wilson P W, Castelli W P, Kannel W B. Coronary risk prediction in 
adults (the Framingham Heart Study). Am J Cardiol, 1987, 59: 91G-94G

13 O'Donnell C J, Cupples L A, D'Agostino R B, et al. Genome-wide association study for subclinical atherosclerosis in major arterial territories in the NHLBI's Framingham Heart Study. BMC Med Genet, 2007, 8: S4

14 Larson M G, Atwood L D, Benjamin E J, et al. Framingham Heart Study 100K project: genome-wide associations for cardiovascular disease outcomes. BMC Med Genet, 2007, 8: S5

15 Kannel W B, Dawber T R, Kagan A, et al. Factors of risk in the development of coronary heart disease-six year follow-up experience. The Framingham Study. Ann Intern Med, 1961, 55: $33-50$

16 Tobert J A. Lovastatin and beyond: the history of the HMG-CoA reductase inhibitors. Nat Rev Drug Discov, 2003, 2: 517-526

17 Kannel W B. Clinical misconceptions dispelled by epidemiological research. Circulation, 1995, 92: 3350-3360

18 Steinberg D, Avigan J, Feigelson E B. Effects of triparanol (Mer-29) on cholesterol biosynthesis and on blood sterol levels in man. J Clin Invest, 1961, 40: 884-893

19 Kirby T J. Cataracts produced by triparanol. (MER-29). Trans Am Ophthalmol Soc, 1967, 65: 494-543

20 Endo A. The discovery and development of HMG-CoA reductase inhibitors. J Lipid Res, 1992, 33: 1569-1582

21 Alberts A W, Chen J, Kuron G, et al. Mevinolin: a highly potent competitive inhibitor of hydroxymethylglutaryl-coenzyme A reductase and a cholesterol-lowering agent. Proc Natl Acad Sci USA, 1980, 77: $3957-3961$

22 Scandinavian Simvastatin Survival Study Group. Randomised trial of cholesterol lowering in 4444 patients with coronary heart disease: the Scandinavian Simvastatin Survival Study (4S). Lancet, 1994, 344: 1383-1389

23 The Long-Term Intervention with Pravastatin in Ischaemic Disease (LIPID) Study Group. Prevention of cardiovascular events and death with pravastatin in patients with coronary heart disease and a broad range of initial cholesterol levels. N Engl J Med, 1998, 339: $1349-1357$
24 Sacks F M, Pfeffer M A, Moye L A, et al. The effect of pravastatin on coronary events after myocardial infarction in patients with average cholesterol levels. Cholesterol and Recurrent Events Trial investigators. N Engl J Med, 1996, 335: 1001-1009

25 Meier B, Bachmann D, Luscher T. 25 years of coronary angioplasty: almost a fairy tale. Lancet, 2003, 361: 527

26 The world health report. 2004

27 Report on cardivascular disease in China. 2010

28 Guarente L, Picard F. Calorie restriction-the SIR2 connection. Cell, 2005, 120: 473-482

29 Koubova J, Guarente L. How does calorie restriction work? Genes Dev, 2003, 17: 313-321

30 Colman R J, Anderson R M, Johnson S C, et al. Caloric restriction delays disease onset and mortality in rhesus monkeys. Science, 2009, 325: 201-204

31 Fontana L, Meyer T E, Klein S, et al. Long-term calorie restriction is highly effective in reducing the risk for atherosclerosis in humans. Proc Natl Acad Sci USA, 2004, 101: 6659-6663

32 Bordone L, Guarente L. Calorie restriction, SIRT1 and metabolism: understanding longevity. Nat Rev Mol Cell Biol, 2005, 6: 298-305

33 Canto C, Auwerx J. Caloric restriction, SIRT1 and longevity. Trends Endocrinol Metab, 2009, 20: 325-331

34 Zhang Q J, Wang Z, Chen $\mathrm{H} \mathrm{Z}$, et al. Endothelium-specific overexpression of class III deacetylase SIRT1 decreases atherosclerosis in apolipoprotein E-deficient mice. Cardiovasc Res, 2008, 80: 191-199

35 Zhou S, Chen H Z, Wan Y Z, et al. Repression of P66Shc expression by SIRT1 contributes to the prevention of hyperglycemia-induced endothelial dysfunction. Circ Res, 2011, 109: 639-648

36 Zhang R, Chen $\mathrm{H} \mathrm{Z}$, Liu J J, et al. SIRT1 suppresses activator protein-1 transcriptional activity and cyclooxygenase-2 expression in macrophages. J Biol Chem, 2010, 285: 7097-7110

37 Sokrates Stein S, Lohmann C, Schäfer N, et al. STRT1 decreases Lox-1-mediated foam cell formation in atherogenesis. Eur Heart J, 2010, 31: 2301-2309

38 Li L, Zhang $\mathrm{H} \mathrm{N}$, Chen H Z, et al. SIRT1 acts as a modulator of neointima formation following vascular injury in mice. Circ Res, 2011, 108: 1180-1189

Open Access This article is distributed under the terms of the Creative Commons Attribution License which permits any use, distribution, and reproduction in any medium, provided the original author(s) and source are credited. 